PROCEEDINGS OF THE

AMERICAN MATHEMATICAL SOCIETY

Volume 132, Number 6, Pages 1581-1588

S 0002-9939(03)07250-2

Article electronically published on November 4, 2003

\title{
COHOMOLOGY AND FINITE SUBGROUPS OF PROFINITE GROUPS
}

\author{
PHAM ANH MINH AND PETER SYMONDS
}

(Communicated by Stephen D. Smith)

\begin{abstract}
We prove two theorems linking the cohomology of a pro- $p$ group $G$ with the conjugacy classes of its finite subgroups.

The number of conjugacy classes of elementary abelian $p$-subgroups of $G$ is finite if and only if the ring $H^{*}(G, \mathbb{Z} / p)$ is finitely generated modulo nilpotent elements.

If the ring $H^{*}(G, \mathbb{Z} / p)$ is finitely generated, then the number of conjugacy classes of finite subgroups of $G$ is finite.
\end{abstract}

\section{Pro- $p$ Groups and elementary abelian PRo- $p$ SUbGroups}

Let $G$ be a profinite group. Fix a fundamental system $\mathcal{U}$ of open neighborhoods $U$ of $1 \in G$ such that $\bigcap_{U \in \mathcal{U}} U=1$ and each $U$ is an open normal subgroup of $G$. For convenience, write $V \leq U$ for $V \subset U$ and $U, V \in \mathcal{U}$. For $U \in \mathcal{U}$, set $G_{U}=G / U$. Denote by $\varphi_{U, V}: G_{V} \rightarrow G_{U}$ the projection map with $V \leq U .\left\{G_{U}, \varphi_{U, V}\right\}$ is an inverse system and

$$
G=\lim _{\longleftarrow} G_{U}
$$

Let $\varphi_{U}: G \rightarrow G_{U}, U \in \mathcal{U}$, be the projection map. For a given prime $p$, let $\mathcal{E}$ (resp. $\mathcal{E}_{U}$ ) be the set of finite elementary abelian $p$-subgroups of $G$ (resp. $G_{U}$ ). (A finite elementary abelian $p$-group is one isomorphic to $(\mathbb{Z} / p)^{n}$, for some $n$, possibly 0 .) It is clear that $\varphi_{U}(E)$ and $\varphi_{U, V}(F)$ are elements of $\mathcal{E}_{U}$, for every $E \in \mathcal{E}, F \in \mathcal{E}_{V}, V \leq$ $U$. Denote by $\psi_{U}: \mathcal{E} \rightarrow \mathcal{E}_{U}$ (resp. $\left.\psi_{U, V}: \mathcal{E}_{V} \rightarrow \mathcal{E}_{U}, V \leq U\right)$ the map induced from $\varphi_{U}\left(\right.$ resp. $\left.\varphi_{U, V}\right)$.

Proposition 1. Given $V \in \mathcal{U}$, there exists $W \in \mathcal{U}$ with $W \leq V$ such that, for every $E \in \mathcal{E}_{W}, \psi_{V, W}(E) \in \operatorname{Im} \psi_{V}$.

Proof. Let $F$ be an element of $\mathcal{E}_{V}$ of rank $n$. Notice that if $F \in \operatorname{Im} \psi_{V, U}$ for $U \leq V$, then in fact $F=\psi_{V, U}(E)$ for $E$ of rank $n$. For each open $U \leq V$, let $\mathcal{F}_{U}$ denote the set of homomorphisms $\eta: F \rightarrow G_{U}$ such that $\psi_{V, U} \eta=\operatorname{Id}_{F}$, and consider the inverse system of the $\mathcal{F}_{U}$ under the maps $\psi$.

If all the $\mathcal{F}_{U}$ are nonempty, then, since the inverse limit over a directed system of nonempty finite sets is always nonempty, $F \in \operatorname{Im} \psi_{V}$. Otherwise, let $U_{F} \leq V$ be such that $\mathcal{F}_{U_{F}}$ is empty. Set $W=\bigcap_{F \notin \operatorname{Im} \psi_{V}} U_{F}$.

Received by the editors November 1, 2002 and, in revised form, February 9, 2003.

2000 Mathematics Subject Classification. Primary 20J06, 17B50.

(C)2003 American Mathematical Society 
The following notation will be used. A prime $p$ will be fixed, and all groups will be pro- $p$ groups unless otherwise indicated. For any profinite group $K, H^{*}(K)$ will always denote the (continuous) cohomology of $K$ with coefficients $\mathbb{Z} / p$. Denote by $I(K)$ the ideal of $H^{*}(K)$ consisting of elements that restrict trivially to every elementary abelian $p$-subgroup of $K$, and by $\mathfrak{R}_{K}$ the nilradical of $H^{*}(K)$. Set

$$
H(K)= \begin{cases}H^{e v}(K) & \text { for } p>2, \\ H^{*}(K), & \text { for } p=2,\end{cases}
$$

and let $H^{+}(K)$ be the ideal of $H^{*}(K)$ consisting of elements of positive degrees. We have

Proposition 2. Given $\eta \in I(G)$, there exist $W \in \mathcal{U}$ and $\xi \in I\left(G_{W}\right)$ such that $\left(\varphi_{W}\right)^{*}(\xi)=\eta$.

In particular, if $H^{*}(G)$ is finitely generated, there exists $U \in \mathcal{U}$ such that $I(G) \subset$ $\left(\varphi_{U}\right)^{*}\left(I\left(G_{U}\right)\right)$.

Proof. Let $\eta$ be an element of $I(G)$. Since $H^{*}(G)=\lim H^{*}\left(G_{U}\right)$, there exist $V$ and $\zeta \in H^{*}\left(G_{V}\right)$ such that $\varphi_{V}^{*}(\zeta)=\eta$. Set $\xi=\varphi_{V, W}^{*}(\zeta)$ with $W$ given in Proposition 1. Also $\varphi_{W}^{*}(\xi)=\eta$. We now prove that $\xi \in I\left(G_{W}\right)$.

Let $E$ be an element of $\mathcal{E}_{W}$, and set $K=\psi_{V, W}(E)$. By Proposition 1, there exists $M \in \mathcal{E}$ such that $\psi_{V}(M)=K$. Consider the commutative diagram

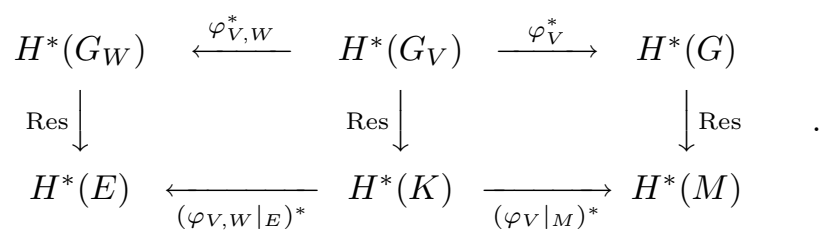

Since $\left.\varphi_{V}\right|_{M}$ is a monomorphism and $\left.\eta\right|_{M}=0$, the right square of the diagram tells us that $\left.\zeta\right|_{K}=0$. So, by the commutativity of the left square, $\left.\xi\right|_{E}=0$. Hence $\xi \in I\left(G_{W}\right)$.

If $H^{*}(G)$ is finitely generated, $V$ can be chosen such that $H^{*}(G)=\operatorname{Im}\left(\varphi_{V}\right)^{*}$. It follows from what we just proved that $I(G) \subset\left(\varphi_{W}\right)^{*}\left(I\left(G_{W}\right)\right)$.

We can now deduce the profinite case of the following theorem of Quillen from the finite case, where many fairly elementary proofs are now known.

Theorem 1 (Quillen [8]). For any profinite group $G, I(G) \subset \mathfrak{R}_{G}$. In other words, every element of $I(G)$ is nilpotent.

Proof. Straightforward from Proposition 2, by noting that $I\left(G_{W}\right) \subset \mathfrak{R}_{G_{W}}$, by the finite case, since $G_{W}$ is finite.

The statement of Quillen's Stratification Theorem involves infinite elementary abelian subgroups, but these are not necessary for the detection result above.

This result also appears in [9].

Proposition 3. Suppose that there exist $U \in \mathcal{U}$ and $a \in G \backslash U$ with $\operatorname{ord}(a)=p$. Set $b=\varphi_{U}(a) \in G_{U}$. Then there exists $\xi \in H^{+}\left(G_{U}\right)$ satisfying:

(a) $\left.\xi\right|_{\langle b\rangle} \neq 0$;

(b) $\left.\varphi_{U}^{*}(\xi)\right|_{\langle a\rangle} \neq 0$. In particular, $\varphi_{U}^{*}(\xi)$ is not nilpotent in $H^{+}(G)$. 
Proof. By [7, Theorem 2.7], there exists $\xi \in H^{+}\left(G_{U}\right)$ such that $\left.\xi\right|_{\langle b\rangle}$ is not nilpotent. From the commutative diagram

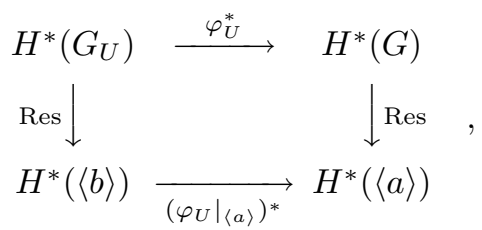

since $H^{*}(\langle b\rangle) \stackrel{\left(\left.\varphi_{U}\right|_{\langle a\rangle}\right)^{*}}{\longrightarrow} H^{*}(\langle a\rangle)$ is an isomorphism, it follows that $\left.\varphi_{U}^{*}(\xi)\right|_{\langle a\rangle}$ is not nilpotent. The proposition follows.

Theorem 2. A pro-p group $A$ is torsion-free if and only if $H^{+}(A) \subset \mathfrak{R}_{A}$.

Proof. If $A$ is not torsion-free, it follows from Proposition 3 that $H^{+}(A)$ contains a non-nilpotent element. If $A$ is torsion-free, then $I(G)=H^{+}(A)$; hence, by Theorem 1 , any element of $H^{+}(A)$ is nilpotent.

Corollary 1. (i) A subgroup $A$ of $G$ is torsion-free if and only $\operatorname{Im}\left(H^{+}(G) \stackrel{\text { Res }}{\longrightarrow}\right.$ $\left.H^{+}(A)\right) \subset \mathfrak{R}_{A}$.

(ii) If $H^{*}(G) / \mathfrak{R}_{G}$ is finitely generated, then $G$ contains an open, normal, torsionfree subgroup $U$.

Proof. (i) If $A$ is torsion-free, it follows from Theorem 2 that $H^{+}(A)$ consists of nilpotent elements; hence any element of $\operatorname{Im}\left(H^{+}(G) \stackrel{\text { Res }}{\rightarrow} H^{+}(A)\right)$ is nilpotent. Conversely, suppose that $A$ contains an element $a$ of order $p$. By Proposition 3 , there exists $\zeta \in H^{+}(G)$ such that $\left.\zeta\right|_{\langle a\rangle}$, hence $\left.\zeta\right|_{A}$, is not nilpotent.

(ii) Suppose that $H^{*}(G) / \Re_{G}$ is finitely generated. Since $H^{*}(G)=\underline{\lim } H^{*}\left(G_{V}\right)$, there exists $U \in \mathcal{U}$ such that $H^{*}\left(G_{U}\right) / \mathfrak{R}_{G_{U}} \stackrel{\left(\varphi_{U}\right)^{*}}{\longrightarrow} H^{*}(G) / \mathfrak{R}_{G}$ is surjective. Hence $\operatorname{Im}\left(H^{+}(G) \stackrel{\text { Res }}{\longrightarrow} H^{+}(U)\right) \subset \mathfrak{R}_{U}$. By $(\mathrm{i}), U$ is torsion-free.

The corollary follows.

Remark 1. The following example shows that the converse of Corollary 1(ii) does not hold. For every $n \in \mathbb{N}$, define $\mathfrak{A}_{n}$ to be the procyclic group $\mathbb{Z}_{2}$ generated by $e_{n}$. Set $\mathfrak{A}=\hat{\bigoplus}_{n} \mathfrak{A}_{n}$ and let $\langle a\rangle \cong \mathbb{Z} / 2$ act on $\mathfrak{A}$ by ${ }^{a} e_{n}=e_{n}^{-1}$. Define $G=\mathfrak{A} \rtimes \mathbb{Z} / 2$. For every $n,\left\langle e_{n} a\right\rangle \cong \mathbb{Z} / 2$ and $\left\langle e_{n} a\right\rangle$ is not conjugate with $\left\langle e_{m} a\right\rangle$ if $m \neq n$. Hence there are infinitely many conjugacy classes of elementary abelian subgroups of $G$. According to Theorem 3 below, $H^{*}(G) / \mathfrak{R}_{G}$ is not finitely generated, although $\mathfrak{A}$ is open, normal and torsion-free in $G$.

We will give a necessary and sufficient condition for $H^{*}(G)$ to be finitely generated as a ring. First we prepare.

Lemma 1. Suppose that $H^{*}(G)$ is finitely generated and $M$ is a finite $\mathbb{F}_{p} G$-module. Then $H^{*}(G, M)$ is Noetherian over $H^{*}(G)$.

Proof. We prove by induction on $n=\operatorname{dim}_{\mathbb{F}_{p}} M$. Suppose that the lemma holds for $n-1$. Consider the exact sequence of $\mathbb{F}_{p} G$-modules

$$
0 \rightarrow \mathbb{F}_{p} \stackrel{f}{\rightarrow} M \stackrel{g}{\rightarrow} N \rightarrow 0
$$


with $N=M / f\left(\mathbb{F}_{p}\right)$. We then have the corresponding long exact sequence of cohomology

$$
\ldots H^{*-1}(G, N) \stackrel{\delta_{*}}{\rightarrow} H^{*}(G) \stackrel{f_{*}}{\rightarrow} H^{*}(G, M) \stackrel{g_{*}}{\rightarrow} H^{*}(G, N) \rightarrow \ldots
$$

By the induction assumption, $H^{*}(G, N)$ is Noetherian over $H^{*}(G)$, hence so is Im $g_{*}$. Pick elements $\xi_{1}, \ldots, \xi_{m}$ of $H^{*}(G, M)$ so that $\left\{g_{*}\left(\xi_{1}\right), \ldots, g_{*}\left(\xi_{m}\right)\right\}$ is a set of generators of $\operatorname{Im} g_{*}$. It follows that $H^{*}(G, M)$ is generated by $\xi_{1}, \ldots, \xi_{m}$, as a module over $\operatorname{Im} f_{*}$. Hence $H^{*}(G, M)$ is Noetherian over $H^{*}(G)$.

Corollary 2. The following are equivalent:

(a) $H^{*}(G)$ is finitely generated;

(b) $G$ contains an open normal, torsion-free subgroup $U$ such that $H^{*}(U)$ is finite;

(c) there exists an open subgroup $K$ of $G$ such that $H^{*}(K)$ is finitely generated;

(d) $H^{*}(K)$ is finitely generated, for any open subgroup $K$ of $G$.

Proof. The implication (b) $\Rightarrow$ (a) was proved by Quillen ([8, Proposition 13.5]) using a spectral sequence argument. It is clear that $(d) \Rightarrow(c)$ and $(d) \Rightarrow(a)$. Suppose that $H^{*}(G)$ is finitely generated and $K$ is open in $G$. By the Eckmann-Shapiro lemma, $H^{*}(K)=H^{*}(G, M)$ with $\left.M=\operatorname{Hom}_{K}\left(\mathbb{F}_{p} G, \mathbb{F}_{p}\right)\right)$. Since $M$ is Noetherian

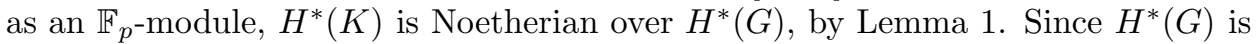
finitely generated, so is $H^{*}(K)$. In particular, if $U$ is given as in Corollary 1 (ii), then $H^{*}(U)$ is finite dimensional, since $H^{+}(U) \subset \mathfrak{R}_{U}$. We then have $(a) \Rightarrow(b)$ and $(a) \Rightarrow(d)$.

Finally, suppose that $K$ is open in $G$ and $H^{*}(K)$ is finitely generated. It follows that $K$ contains an open, normal, torsion-free subgroup $U$ such that $H^{*}(U)$ is finite. Since $U$ is also open in $G, U$ contains an open, normal subgroup $V$ of $G$. Since $V$ is torsion-free and open in $U, H^{*}(V)$ is finite. So $H^{*}(G)$ is finitely generated. The implication $(c) \Rightarrow(a)$ is then proved.

Remark 2. In [8], it was proved that, if $H^{*}(G)$ is finitely generated, then $G$ has only finitely many conjugacy classes of elementary abelian $p$-subgroups. However, the converse does not hold: the group $\mathfrak{A}$ given above has only one elementary abelian subgroup, which is the one of rank 0 , while $H^{*}(\mathfrak{A})$ is an exterior algebra with an infinite number of generators of degree 1 , hence is not finitely generated.

From now on, fix $\mathcal{F}$ a set of representatives of conjugacy classes of elementary abelian $p$-subgroups of $G$. We now give a cohomological criterion for $\mathcal{F}$ to be finite, as follows.

Theorem 3. The following are equivalent:

(a) There exists an open normal subgroup $A$ of $G$ such that $E A \neq F A$, for $E \neq F$ in $\mathcal{F}$

(b) $H^{*}(G) / \mathfrak{R}_{G}$ is finitely generated (as a ring);

(c) $\mathcal{F}$ is finite.

Proof. (a) $\Rightarrow$ (c): Let $\mathcal{S}$ be the set of subgroups of $G_{A}$. The map $f: \mathcal{F} \rightarrow \mathcal{S}, E \mapsto$ $E A / A$ is then injective. Since $f$ maps $\mathcal{F}$ injectively into the finite set $\mathcal{S}, \mathcal{F}$ is finite.

The implication (c) $\Rightarrow$ (a) is clear. We now prove (b) $\Rightarrow$ (c). Suppose that $H^{*}(G) / \mathfrak{R}_{G}$ is finitely generated. Let $U$ be the open, normal, torsion-free subgroup 
of $G$ as given in Corollary 1(ii). It follows from the proof of the corollary that, for every $V \leq U, V$ is torsion-free and $H^{*}\left(G_{V}\right) / \mathfrak{R}_{G_{V}} \stackrel{\left(\varphi_{V}\right)^{*}}{\rightarrow} H^{*}(G) / \mathfrak{R}_{G}$ is surjective.

Let $\mathcal{M}$ be a set of representatives of conjugacy classes of maximal elementary abelian pro- $p$ subgroups of $G$ and let $E, F$ be two different elements of $\mathcal{M}$. Since $U$ is torsion-free, it follows that $\varphi_{V}$ maps $E$ (resp. $F$ ) isomorphically to $\varphi_{V}(E)$ (resp. $\varphi_{V}(F)$ ), for every $V \leq U$. Furthermore, since $E \neq F$, there exists $W \leq U$ such that each of $\varphi_{W}(E), \varphi_{W}(F)$ is not conjugate (in $G_{W}$ ) to any subgroup of the other. According to [7, Theorem 2.7], there exist $\xi, \eta \in H^{*}\left(G_{W}\right)$ such that $\left.\xi\right|_{\varphi_{W}(E)},\left.\eta\right|_{\varphi_{W}(F)}$ are not nilpotent, and $\left.\xi\right|_{\varphi_{W}(F)}=0,\left.\eta\right|_{\varphi_{W}(E)}=0$. Set $\xi^{\prime}=\left(\varphi_{W}\right)^{*}(\xi), \eta^{\prime}=\left(\varphi_{W}\right)^{*}(\eta)$. It follows that $\left.\xi^{\prime}\right|_{E},\left.\eta^{\prime}\right|_{F}$ are not nilpotent, and $\left.\xi^{\prime}\right|_{F}=0,\left.\eta^{\prime}\right|_{E}=0$; in particular, $\xi^{\prime}$ and $\eta^{\prime}$ are not nilpotent. Since $H^{*}\left(G_{U}\right) / \mathfrak{R}_{G_{U}} \stackrel{\left(\varphi_{U}\right)^{*}}{\longrightarrow} H^{*}(G) / \mathfrak{R}_{G}$ is surjective, there exist $\zeta, \theta \in H^{*}\left(G_{U}\right)$ such that $\left.\zeta\right|_{\varphi_{U}(E)},\left.\theta\right|_{\varphi_{U}(F)}$ are not nilpotent, and $\left.\zeta\right|_{\varphi_{U}(F)}=0,\left.\theta\right|_{\varphi_{U}(E)}=0$. Also, by [7, Theorem 2.7], it follows that each of $\varphi_{U}(E), \varphi_{U}(F)$ is not conjugate to any subgroup of the other; in particular, $\varphi_{U}(E) \neq \varphi_{U}(F)$. So $\psi_{U}$ maps $\mathcal{M}$ injectively into $\mathcal{E}_{U}$. Hence $\mathcal{M}$ is finite, and so is $\mathcal{F}$.

Finally, let us prove $(\mathrm{c}) \Rightarrow(\mathrm{b})$. Suppose that $\mathcal{F}$ is finite. It follows that there exists $T \in \mathcal{U}$ such that, for every $E \in \mathcal{E},\left.\varphi_{T}\right|_{E}$ is an isomorphism, and $\psi_{T}$ maps $\mathcal{F}$ injectively into $\mathcal{E}_{T}$. For every $E \in \mathcal{F}$, the restriction map $\operatorname{Res}_{\varphi_{T}(E)}^{G_{T}}$ induces an action of $H\left(G_{T}\right)$ on $H^{*}\left(\varphi_{T}(E)\right)$, hence on $H^{*}(E)$; furthermore, by [4, Corollary 7.4.7], $H^{*}(E)$ is a finitely generated $H\left(G_{T}\right)$-module. Besides, the inflation $\left(\varphi_{T}\right)^{*}$ also induces an action of $H\left(G_{T}\right)$ on $H^{*}(G)$ so that $\operatorname{Res}_{E}^{G}$ is an $H\left(G_{T}\right)$ homomorphism, for every $E \in \mathcal{F}$. Set $\mathfrak{J}=\prod_{E \in \mathcal{F}} H^{*}(E)$. Since $\mathcal{F}$ is finite, $\mathfrak{J}$ is a finitely generated $H\left(G_{T}\right)$-module. Since $H^{*}(G) / I(G)$ is isomorphic to a submodule of $\mathfrak{J}$, it is also a finitely generated $H\left(G_{T}\right)$-module. Furthermore, $I(G) \subset \mathfrak{R}_{G}$ implies that $H^{*}(G) / \mathfrak{R}_{G}$ is, in turn, a finitely generated $H\left(G_{T}\right)$-module. Since $H\left(G_{T}\right)$ is a finitely generated algebra, so is $H^{*}(G) / \mathfrak{R}_{G}$.

It has been pointed out to us by Henn that such a result can also be deduced from material in $[6]$.

Let $\mathcal{E}$ be the category with objects the elementary abelian $p$-subgroups of $G$ and with morphisms from $A$ to $B$ defined to be the homomorphisms $\theta: A \rightarrow B$ of the form $\theta a=g a g^{-1}$ for some $g \in G$. Set $\mathfrak{L}={\underset{\leftarrow \in \mathcal{E}}{\lim }}_{E} H(E)$. It is clear that the product of restriction maps

$$
\mathfrak{L} \rightarrow \mathfrak{J}=\prod_{E \in \mathcal{F}} H^{*}(E)
$$

is a monomorphism of $H(G)$-modules. From the proof of the above theorem, if $\mathcal{F}$ is finite, then $\mathfrak{J}$ is Noetherian over $H(G)$. Thus $\mathfrak{L}$ is finitely generated over $H\left(G_{T}\right)$ and hence over $H(G)$. We now have

Proposition 4. If $\mathcal{F}$ is finite, then $\mathfrak{L}$ is finitely generated over $H(G)$.

According to [8, Proposition 13.4], if $\mathcal{F}$ is finite, the map

$$
H(G) \stackrel{\operatorname{Res}^{G}}{\rightarrow} \mathfrak{L}
$$

is an $F$-isomorphism. In other words, given $x \in I(G)$ and $y \in \mathfrak{L}$, there exists an integer $n=n_{x, y}$ such that $x^{n}=0$ and $y^{p^{n}} \in \operatorname{Im} \operatorname{Res}^{G}$. We now give a sufficient 
condition for $\operatorname{Res}^{G}$ to be a uniform $F$-isomorphism (i.e., the integer $n$ can be chosen independently of $x$ and $y$ ).

Theorem 4. If $H^{*}(G)$ is finitely generated, then $\operatorname{Res}^{G}$ is a uniform F-isomorphism.

Proof. Suppose that $H^{*}(G)$ is finitely generated. $\mathcal{F}$ is then finite and the map $H(G) \stackrel{\operatorname{Res}^{G}}{\longrightarrow} \mathfrak{L}$ is an $F$-isomorphism. By Proposition $4, \mathfrak{L}$ is finitely generated over $H(G)$. So there exists an integer $r$ such that $y^{p^{r}} \in \operatorname{Im} \operatorname{Res}^{G}$, for every $y \in \mathfrak{L}$.

By Proposition 2, there exists $U \in \mathcal{U}$ such that $I(G) \subset\left(\varphi_{U}\right)^{*}\left(I\left(G_{U}\right)\right)$. Since $G_{U}$ is finite, there exists an integer $s$ such that $x^{s}=0$, for every $x \in I(G)$. The theorem is then proved, by setting $n=n_{x, y}=\max (r, s)$.

\section{PRO- $p$ GROUPS AND FINITE $p$-SUBGROUPS}

Let $G$ be a pro- $p$ group. The purpose of this section is to prove the following.

Theorem 5. If $H^{*}(G)$ is finitely generated, then $G$ has only finitely many conjugacy classes of finite p-subgroups.

An analogous result is known for discrete groups of type vFP (see [1], IX, 13.2) and for analytic pro- $p$ groups (where the hypothesis is vacuous, see [2]).

Our proof depends on some deep results on unstable algebras over the Steenrod algebra.

We will need:

Lemma 2. If $H^{*}(G)$ is finitely generated and $C$ is a finite central subgroup of $G$, then $H^{*}(G / C)$ is finitely generated.

Proof. Without loss of generality, we may suppose that $C$ is of order $p$. Write $K=G / C$, and let $z \in H^{2}(K)$ be the cohomology class classifying the central extension

$$
1 \rightarrow C \rightarrow G \rightarrow K \rightarrow 1 \text {. }
$$

There exists then an open, normal subgroup $L$ of $K$ such that $\operatorname{Res}_{L}^{K}(z)=0$. Therefore, the preimage $H$ of $L$ in $G$ is isomorphic to $C \times L$. In other words, $L$ can be considered as an open subgroup of $G$. By Corollary $2, H^{*}(L)$, and so $H^{*}(K)$, are finitely generated.

For every subgroup $P$ of $G$, denote by $N_{G}(P)$ (resp. $\left.C_{G}(P)\right)$ the normalizer (resp. centralizer) of $P$ in $G$. We have

Lemma 3. If $P$ is a subgroup of $G$ of order $p$, then:

(i) $N_{G}(P)=C_{G}(P)$;

(ii) $H^{*}\left(C_{G}(P)\right)$ and $H^{*}\left(C_{G}(P) / P\right)$ are finitely generated, provided that so is $H^{*}(G)$.

Proof. (i) follows from the fact that $N_{G}(P) / C_{G}(P)$ is embedded into $\operatorname{Aut}(P)$, which is of order $p-1$.

(ii) By Lemma 2, we need only prove that $H^{*}\left(C_{G}(P)\right)$ is finitely generated. By Corollary 2, there exists $U$ open, normal, torsion-free in $G$. Set $K=\langle U, P\rangle$. $K$ is then of $p$-rank 1 (i.e., every elementary abelian subgroup of $K$ is of rank at most 1 ), open in $G$ and has finitely generated cohomology, by Corollary 2. Furthermore, $C_{K}(P)$ is open in $C_{G}(P)$. By Corollary 2, it suffices to prove that $H^{*}\left(C_{K}(P)\right)$ is finitely generated. According to the theory in [3] (see also [6, Corollary 1.7]) the 
unstable algebra $T^{V} H^{*}(G)$ is Noetherian and also $T^{V} H^{*}(G)=\prod_{(\rho)} H^{*}\left(C_{K}(\operatorname{im} \rho)\right)$, where the product is taken over conjugacy classes of homomorphisms $\rho: \mathbb{Z} / p \rightarrow K$. It follows that $H^{*}\left(C_{K}(P)\right)$ is finitely generated.

Proof of Theorem 5. Since $H^{*}(G)$ is finitely generated, it follows from Corollary 2 that there exists an open normal, torsion-free subgroup $U$ of $G$. Define

$n_{G}=\min \left\{n|| G / U \mid=p^{n}\right.$ for some open, normal, torsion-free subgroup $U$ of $\left.G\right\}$

We argue by induction on $n_{G}$. If $n_{G}=1$, the conclusion follows, since every finite subgroup of $G$ is elementary abelian of rank 1 . Suppose that the theorem holds if $n_{G}<m$.

Assume that $n_{G}=m$. It is known that the number of conjugacy classes of elementary abelian $p$-subgroups of rank 1 of $G$ is finite. Let $\left\{C_{1}, \ldots, C_{k}\right\}$ be a set of representatives of such conjugacy classes. It is clear that, for any finite $p$ subgroup $P$ of $G$, there exist $g \in G$ and $i$ such that $P^{g}$ contains $C_{i}$ as a central subgroup. Let $\mathcal{N}_{i}$ be the set of finite subgroups of $G$ containing $C_{i}$ as a central subgroup, $1 \leq i \leq k$. It is then sufficient to prove that the number of conjugacy classes in $\mathcal{N}_{i}$ is finite, $1 \leq i \leq k$.

Fix such an $i$. Note that $\mathcal{N}_{i}$ coincides with the set of finite subgroups of $K=$ $C_{G}\left(C_{i}\right)$ containing $C_{i}$, hence is in one-to-one correspondence with the set of finite subgroups of $H=C_{G}\left(C_{i}\right) / C_{i}$. Therefore, we need only prove that $H$ has many finite conjugacy classes of finite subgroups. Let $U$ be open, normal, torsion-free in $G$ with $|G / U|=p^{n_{G}}$ and set $V=U \cap K . V$ is then open, normal, torsion-free in $K$ and $|K / V| \leq|G / U|=p^{n_{G}}$. So $n_{K} \leq n_{G}$. Since $n_{H}=n_{K}-1<n_{G}$, it follows from the induction hypothesis that $H$ has many finite conjugacy classes of finite subgroups. The theorem is proved.

\section{REFERENCES}

1. K. S. Brown, Cohomology of groups, Graduate Texts in Mathematics, vol. 87, Springer-Verlag, Berlin, 1982. MR 83k:20002

2. J. D. Dixon, M.P.F. du Sautoy, A. Mann, and D. Segal, Analytic pro-p groups (2nd edition), London Mathematical Society Lecture Note Series, vol. 157, Cambridge University Press, Cambridge, 1999. MR 94e:20037

3. W. G. Dwyer and C. Wilkerson, Smith Theory and the functor T, Comment. Math. Helvetici 66 (1991), 1-17. MR 92i:55006

4. L. Evens, The cohomology of groups, Oxford Mathematical Monographs, Clarendon Press, 1991. MR 93i:20059

5. H.-W. Henn, Centralizers of elementary abelian p-subgroups and mod-p cohomology of profinite groups, Duke Math. J. 91 (1998), 561-585. MR 99b:20083

6. H.-W. Henn, J. Lannes, and L. Schwarz, The categories of unstable modules and unstable algebras over the Steenrod algebra modulo nilpotent objects, Amer. Jour. Math. 115 (1993), 1053-1106. MR 94i:55024

7. D. Quillen, A cohomological criterion for p-nilpotence, J. Pure Appl. Algebra 1 (1971), 361-372. MR 47:6886 
8. D. Quillen, The spectrum of an equivariant cohomology ring II, Ann. Math. 94 (1971), 573-602. MR 45:7743

9. C. Scheiderer, Farrell cohomology and Brown theorems for profinite groups, Manuscripta Math. 91 (1996), 247-281. MR 97j:20050

Department of Mathematics, College of Science, University of Hue, Dai hoc Khoa hoc, Hue, Vietnam

E-mail address: paminh@dng.vnn.vn

Current address: Inst. Hautes Études Sci., Le Bois-Marie, 35 Route de Chartres, F-91440 Bures-sur-Yvette, France

Department of Mathematics, U.M.I.S.T., P.O. Box 88, Manchester M60 1QD, England

E-mail address: Peter.Symonds@umist.ac.uk 